\title{
Three-Dimensional Virtual Bone Bank System Workflow for Structural Bone Allograft Selection: A Technical Report
}

\author{
Lucas Eduardo Ritacco, ${ }^{1,2}$ German Luis Farfalli, ${ }^{2}$ Federico Edgardo Milano, \\ Miguel Angel Ayerza, ${ }^{2}$ Domingo Luis Muscolo, ${ }^{2}$ and Luis Aponte-Tinao ${ }^{2}$ \\ ${ }^{1}$ Virtual Planning and Navigation Unit, Department of Health Informatics, Italian Hospital of Buenos Aires, \\ 1199 Buenos Aires, Argentina \\ ${ }^{2}$ Institute of Orthopedics "Carlos E. Ottolenghi", Italian Hospital of Buenos Aires, Potosí 4247, 1199 Buenos Aires, Argentina \\ Correspondence should be addressed to Lucas Eduardo Ritacco; lucas.ritacco@hospitalitaliano.org.ar
}

Received 15 February 2013; Accepted 19 March 2013

Academic Editor: Andreas Leithner

Copyright (C) 2013 Lucas Eduardo Ritacco et al. This is an open access article distributed under the Creative Commons Attribution License, which permits unrestricted use, distribution, and reproduction in any medium, provided the original work is properly cited.

Structural bone allograft has been used in bone defect reconstruction during the last fifty years with acceptable results. However, allograft selection methods were based on 2-dimensional templates using X-rays. Thanks to preoperative planning platforms, threedimensional (3D) CT-derived bone models were used to define size and shape comparison between host and donor. The purpose of this study was to describe the workflow of this virtual technique in order to explain how to choose the best allograft using a virtual bone bank system. We measured all bones in a 3D virtual environment determining the best match. The use of a virtual bone bank system has allowed optimizing the allograft selection in a bone bank, providing more information to the surgeons before surgery. In conclusion, 3D preoperative planning in a virtual environment for allograft selection is an important and helpful tool in order to achieve a good match between host and donor.

\section{Introduction}

The uses of bone allograft after bone tumor resection have been described with acceptable results in osteoarticular, transepiphyseal, and intercalary reconstructions [1-4].

Selection of the closest anatomical match between the host and the donor is crucial in order to obtain adequate joint stability, alignment, appropriate wound closure, and minor degenerative changes of the articular surface in osteoarticular allograft.

Since 1950, bone allograft selection according to size and shape for limb reconstruction was made by comparing Xray images between donors and patient [5]. This method had inaccuracies between the X-ray magnification scale and real bone, altering the final selection [6]. In the 1970s, CT scanner allowed to refine these inaccuracies taking into account one image slice in two dimensions [7]. The previous two decades have seen an increase in the use of virtual scenarios and informatics developments for preoperative planning [8-10].
Three-dimensional patient-specific anatomical models can be constructed from medical image data.

We described a virtual technique capable of selecting a suitable allograft according to size and shape through a threedimensional virtual model.

The aim of this paper was to describe the workflow of this technique in different cases in order to explain how to choose the best allograft using a virtual bone bank system.

\section{Material and Methods}

Three-dimensional (3D) virtual bone models from host and donor were obtained following these steps: image acquisition, image segmentation, and $3 D$ bone reconstruction, described in detail below.

Once this workflow was completely defined, we were capable of measuring each bone in a virtual environment and establishing 3D comparisons between host and donor to determine the best match. 


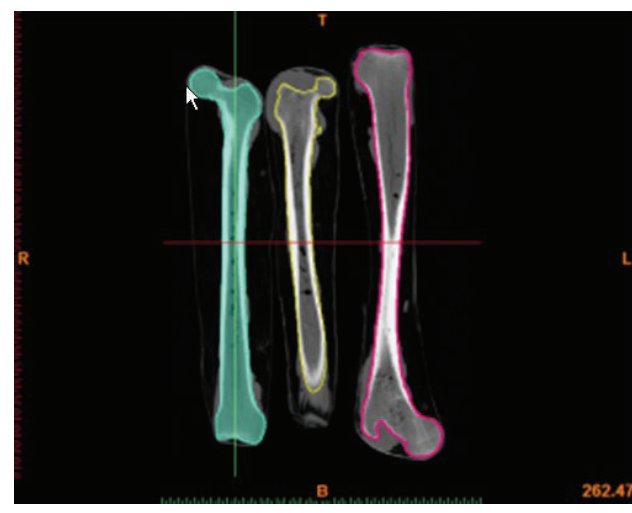

(a)

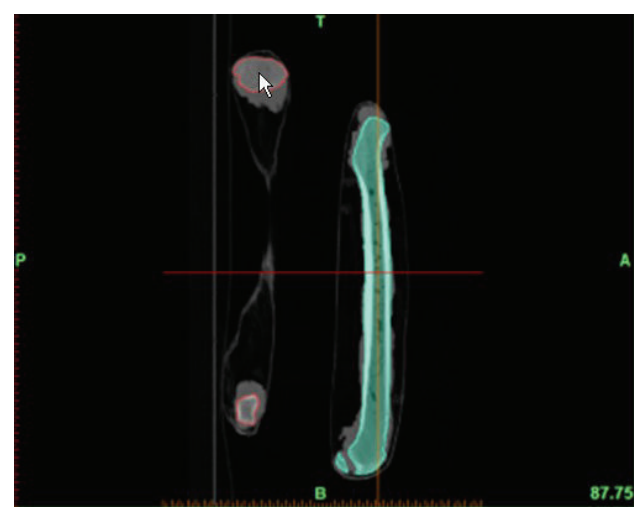

(c)

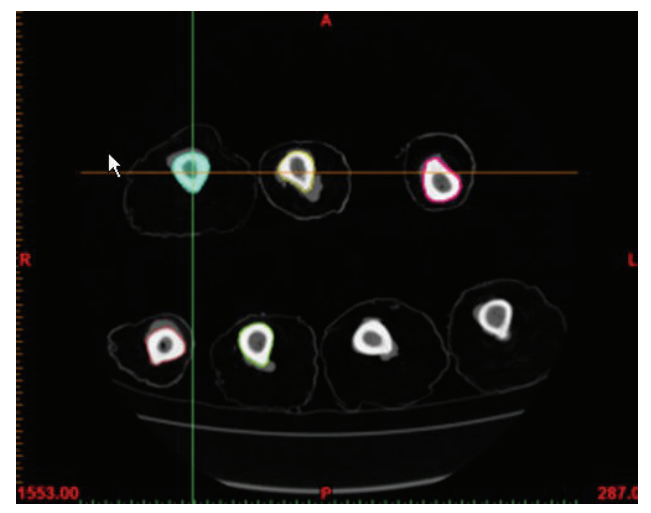

(b)

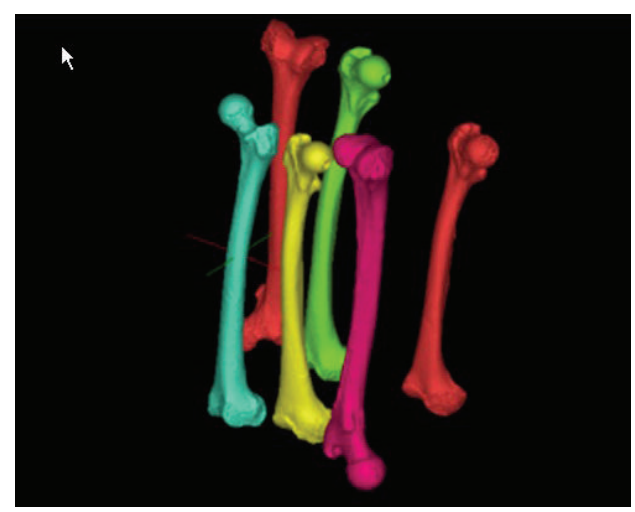

(d)

Figure 1: (a)-(c) Image segmentation. (d) Bone allograft was 3D reconstructed.

All images were acquired using a CT scanner (Mutislice 64, Aquilion, Toshiba Medical Systems, Otawara, Japan). Magnified slices with $0.5 \mathrm{~mm}$ thickness were obtained using a soft tissue standard filter, a matrix of $512 \times 512$ pixels, and stored in Digital Imaging and Communication in Medicine (DICOM) format.

In order to establish comparable measurements, image acquisition protocols should be equal in host and donor. Image magnification process is an important step to obtain images in high definition. Thus, we suggest magnifying the image as much as possible.

Image segmentation is a process which consists in changing the representation of a DICOM image into an image that is easier to analyze. We used a specialized software for the segmentation tasks (Mimics software, Leuven, Belgium). Through this process, an operator assigns a color to every pixel in an image such that pixels with the same intensity define a separate structure: for example, cortical and trabecular bone. In our case, the bone after the segmentation process was isolated from the other tissues and structures such as muscle, fat, skin, ice, and metal table of CT scanner (Figure 1).

The result of image segmentation is a set of segments or a set of contours extracted from the image that collectively cover the entire 3D bone model. Each of the pixels in a region is similar according to intensity. The resulting contours after image segmentation were used to create $3 \mathrm{D}$ reconstructions with the help of interpolation algorithms. In this manner, a three-dimensional bone model was created (Figure 1). The segmentation process of a whole large bone takes a mean of 10 hours.

Take into account that the "contrast" in the CT for image segmentation is the calcium density. In oncologic patients, pain and lack of mobility lead to low calcium density. In consequence, the cortical and trabecular bones are replaced by the tumor action, erasing the anatomical shape and recognizable landmarks. In this way, $3 \mathrm{D}$ tumoral bone models appear to be incomplete.

Hereby, exploiting the symmetry of the human body [11, 12 ], we create a $3 \mathrm{D}$ mirror model from the patient's healthy side.

In order to select the best size, six anatomical landmarks were defined determining three principal measures from the $3 \mathrm{D}$ mirror model: $\mathrm{A}$ is transepicondylar, $\mathrm{B}$ is medial anterior-posterior condyle, and $\mathrm{C}$ is lateral anterior-posterior condyle (Figure 2). Once the whole bank was measured we created a table with all the $\mathrm{ABC}$ extents. First we search, as a screening step, $\mathrm{ABC}$ donors closest to $\mathrm{ABC}$ host. Next, in order to compare and select the best shape, 3D mirror model is overlapped with the available donors. Before comparing $3 \mathrm{D}$ shapes, all 3D bones were positioned in the same coordinate system. This process is called $3 \mathrm{D}$ registration. 


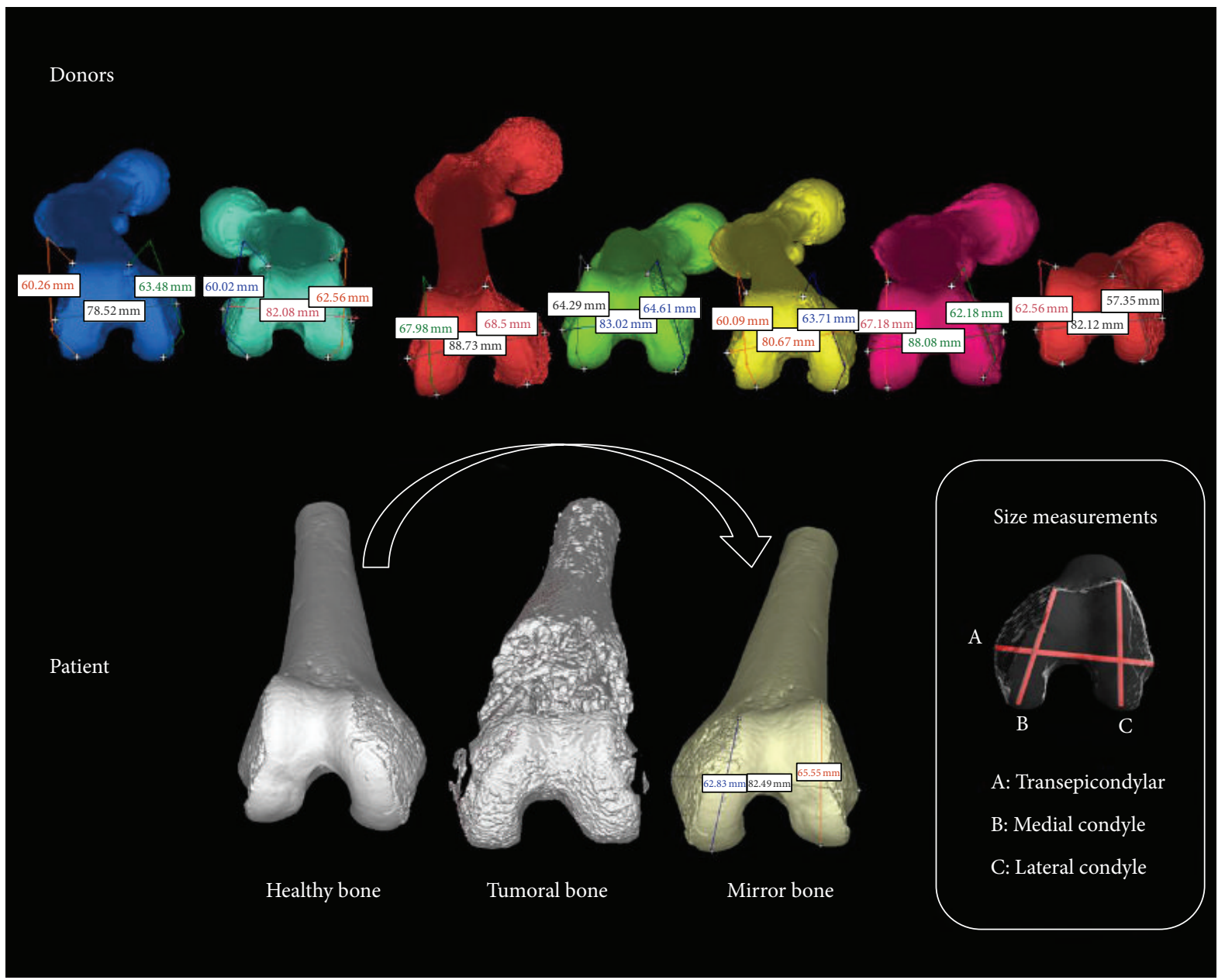

FIgURE 2: Donors were measured using $\mathrm{ABC}$ measurements. The healthy bone of the patient was mirrored and then measured with $\mathrm{ABC}$ measures in order to compare the best match according to the sizes.

Using a point cloud model of surfaces, it was possible to obtain a numeric value (a mean) that reflects the goodness of the match [12].

Distances between host and donor were illustrated in a colorimetric mapping.

This tool allowed us to determine which is the most similar area with a color scale (Figure 3 ).

Since it is not an easy task to determine natural landmarks in transepiphyseal and diaphyseal allografts, only it is possible to determine a match by overlapping the host with available donors (Figure 4).

\section{Discussion}

Paul et al. in their study [11] explored the use of 2-dimensional template comparison for allograft matching. However, the cited study also describes a 3D registration method and states that the 2-dimensional template comparison is ineffective.

The correspondence between the 3D models and the real bone depends heavily on CT scanner, segmentation, and interpolation software [11].
Published studies on three-dimensional preoperative planning using virtual environments for allograft selection have reported benefits in pelvis and femur [11-14]. The use of this technology allowed optimizing the allograft selection in a bone bank, providing three-dimensional visual information to the surgeon before surgery is executed.

As well, if the host has to be compared against multiple available donors, the process would be very time consuming if it were to be performed manually [14]. Actually, the algorithms described in the cited articles were capable of automatically choosing the best allograft according to the size and shape criteria. We also have already published acceptable results applying an automatic method to select the best match $[15,16]$.

Although we know that anatomical matching is only one of multiple factors that could affect the outcome of an allograft reconstruction, poor matching between host and donor can alter joint kinematics and load distribution, leading to bone resorption and joint degeneration [17, 18]. Pathological studies showed that allografts retrieved from patients with a nonsimilar joint had earlier and more advanced degenerative 

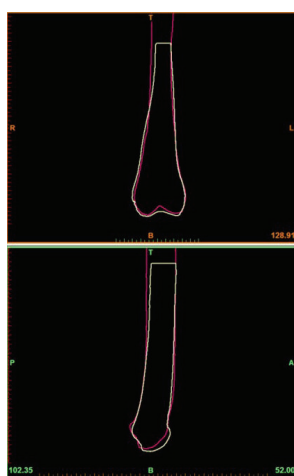

Host-donor overlapping

(a)
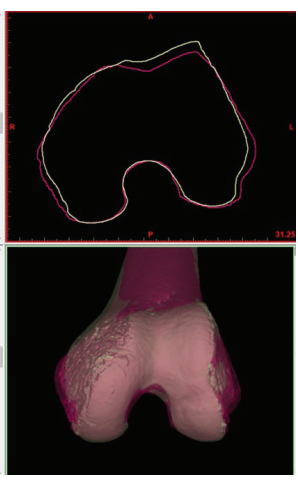

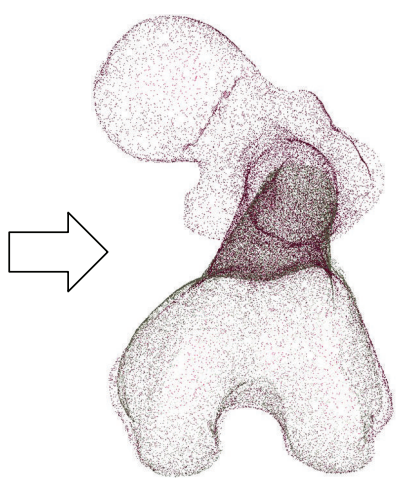

Point cloud data

(b)

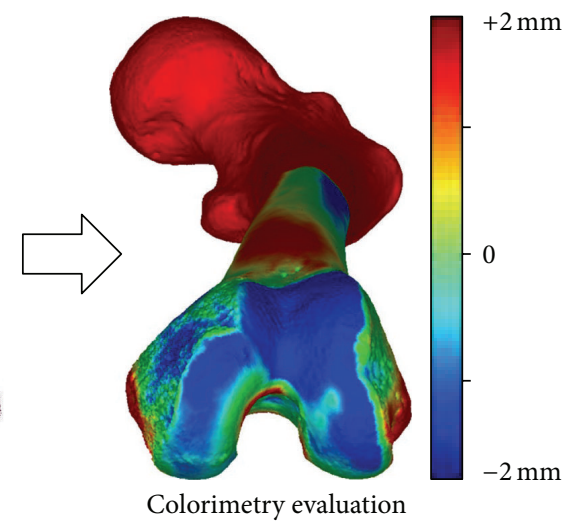

(c)

FIGURE 3: (a) Host and donor were overlapped in a virtual platform in order to compare the best match according to the shapes. (b) 3D models were exported to point cloud data. (c) A colorimetry evaluation was applied comparing host and donor surfaces.

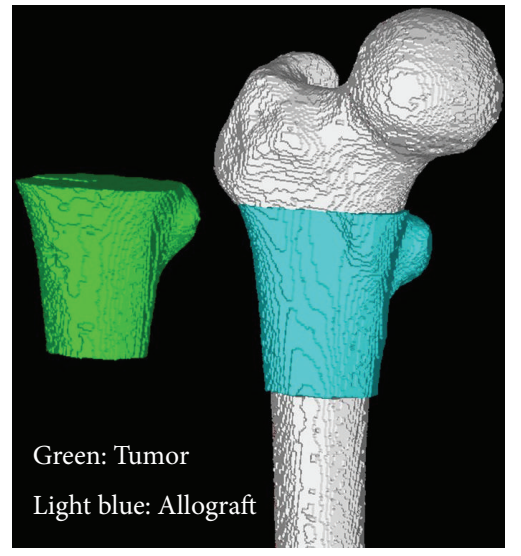

(a)

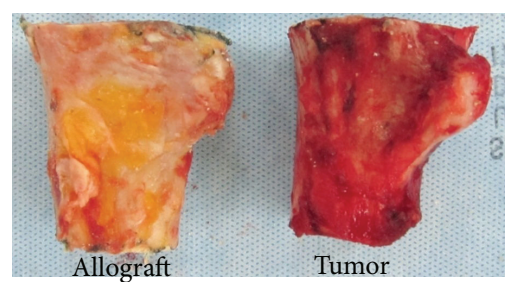

(b)

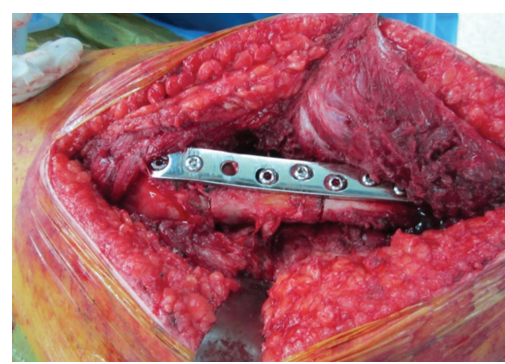

(c)

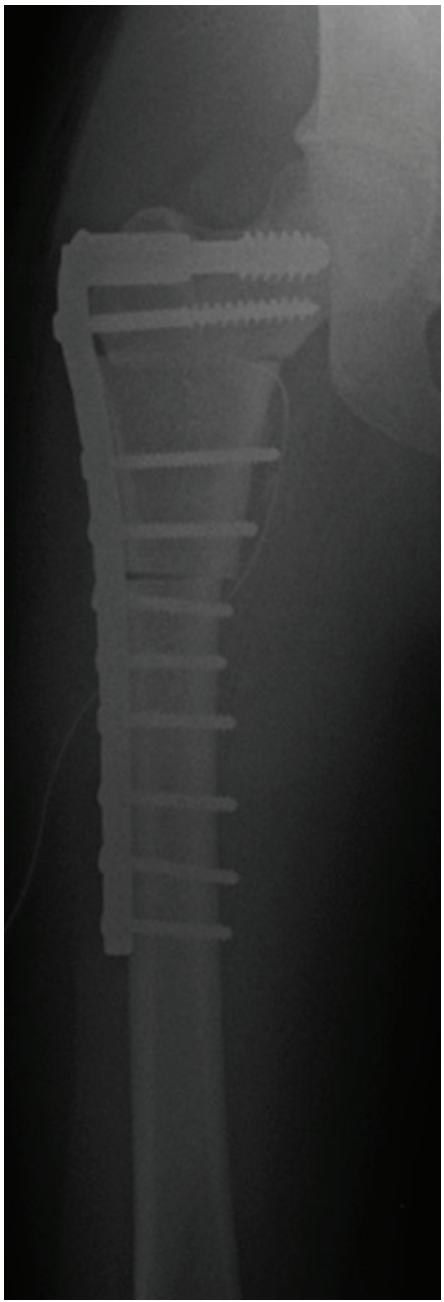

(d)

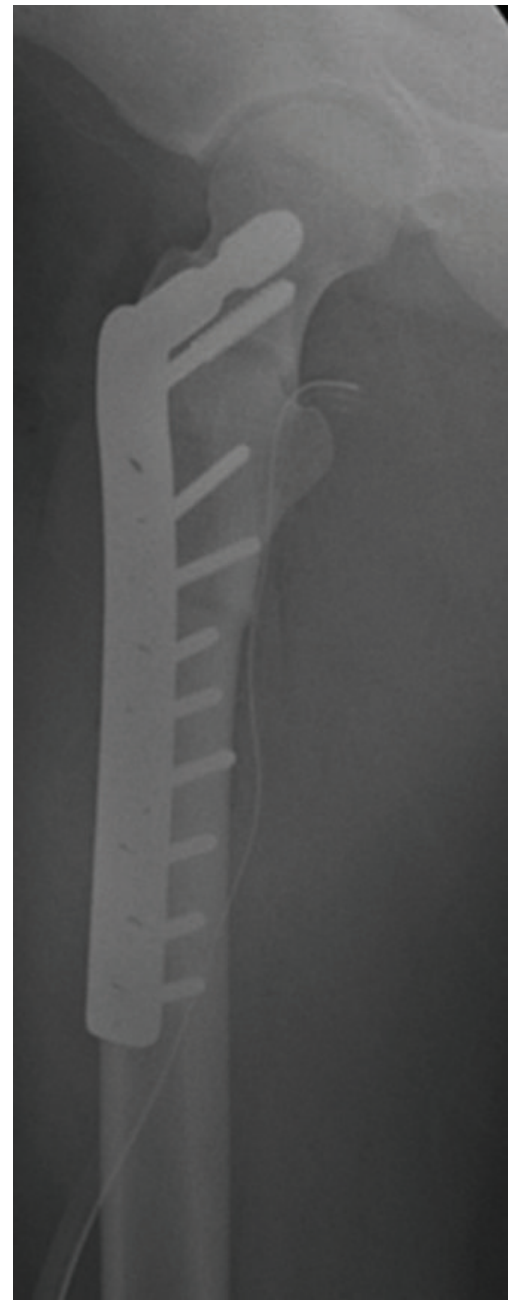

(e)

Figure 4: (a) Bone allograft was selected according to the shapes comparison between host and donor. The original tumor diagnosis was a chondrosarcoma. (b) Allograft was selected and tumor was resected. (c) Allograft was fixed in the patient through a plate and screws. (d) and (e) Postoperative X-rays. 
changes in the articular cartilage than did allografts retrieved from patients with a stable joint $[17,19]$.

\section{Conclusion}

We consider that a three-dimensional preoperative planning in a virtual environment for allograft selection is an important and helpful tool in order to achieve a good match between host and donor. Currently, we are following these patients to assess their limb function at several postoperative intervals.

\section{References}

[1] D. L. Muscolo, M. A. Ayerza, L. A. Aponte Tinao, and M. Ranalletta, "Distal femur osteoarticular allograft reconstruction after grade III open fractures in pediatric patients," Journal of Orthopaedic Trauma, vol. 18, no. 5, pp. 312-315, 2004.

[2] H. J. Mankin, M. C. Gebhardt, L. C. Jennings, D. S. Springfield, and W. W. Tomford, "Long-term results of allograft replacement in the management of bone tumors," Clinical Orthopaedics and Related Research, no. 324, pp. 86-97, 1996.

[3] H. J. Mankin, M. C. Gebhardt, and W. W. Tomford, “The use of frozen cadaveric allografts in the management of patients with bone tumors of the extremities," Orthopedic Clinics of North America, vol. 18, no. 2, pp. 275-289, 1987.

[4] D. L. Muscolo, M. A. Ayerza, L. A. Aponte-Tinao, and M. Ranalletta, "Partial epiphyseal preservation and intercalary allograft reconstruction in high-grade metaphyseal osteosarcoma of the knee," Journal of Bone and Joint Surgery A, vol. 87, supplement 1, part 2, pp. 226-236, 2005.

[5] C. E. Ottolenghi, "Massive osteoarticular bone grafts. Transplant of the whole femur," Journal of Bone and Joint Surgery, British, vol. 48, no. 4, pp. 646-659, 1966.

[6] K. S. Conn, M. T. Clarke, and J. P. Hallett, "A simple guide to determine the magnification of radiographs and to improve the accuracy of preoperative templating," Journal of Bone and Joint Surgery, British, vol. 84, no. 2, pp. 269-272, 2002.

[7] D. L. Muscolo, M. A. Ayerza, L. A. Aponte-Tinao, and M. Ranalletta, "Use of distal femoral osteoarticular allografts in limb salvage surgery. Surgical technique," The Journal of Bone and Joint Surgery, vol. 88, supplement 1, part 2, pp. 305-321, 2006.

[8] E. Y. Chao, P. Barrance, E. Genda, N. Iwasaki, S. Kato, and A. Faust, "Virtual reality (VR) techniques in orthopaedic research and practice," Studies in Health Technology and Informatics, vol. 39, pp. 107-114, 1997.

[9] D. Cheong and G. D. Letson, "Computer-assisted navigation and musculoskeletal sarcoma surgery," Cancer Control, vol. 18, no. 3, pp. 171-176, 2011.

[10] M. Robiony, I. Salvo, F. Costa et al., "Accuracy of virtual reality and stereolithographic models in maxillo-facial surgical planning," Journal of Craniofacial Surgery, vol. 19, no. 2, pp. 482489, 2008.

[11] L. Paul, P. L. Docquier, O. Cartiaux, O. Cornu, C. Delloye, and $\mathrm{X}$. Banse, "Selection of massive bone allografts using shapematching 3-dimensional registration," Acta Orthopaedica, vol. 81, no. 2, pp. 252-257, 2010.

[12] L. E. Ritacco, A. A. Espinoza Orías, L. Aponte-Tinao, D. L. Muscolo, F. G. de Quirós, and I. Nozomu, “Three-dimensional morphometric analysis of the distal femur: a validity method for allograft selection using a virtual bone bank," Studies in Health Technology and Informatics, vol. 160, part 2, pp. 1287-1290, 2010.

[13] L. Paul, P. L. Docquier, O. Cartiaux, O. Cornu, C. Delloye, and X. Banse, "Inaccuracy in selection of massive bone allograft using template comparison method," Cell and Tissue Banking, vol. 9, no. 2, pp. 83-90, 2008.

[14] H. Bou Sleiman, L. Paul, L.-P. Nolte, and M. Reyes, "Comparative evaluation of pelvic allograft selection methods," Annals of Biomedical Engineering, 2013.

[15] H. Bou Sleiman, L. E. Ritacco, L. Aponte-Tinao, D. L. Muscolo, L. P. Nolte, and M. Reyes, "Allograft selection for transepiphyseal tumor resection around the knee using three-dimensional surface registration," Annals of Biomedical Engineering, vol. 39, no. 6, pp. 1720-1727, 2011.

[16] L. E. Ritacco, C. Seiler, G. L. Farfalli et al., "Validity of an automatic measure protocol in distal femur for allograft selection from a three-dimensional virtual bone bank system," Cell and Tissue Banking, pp. 1-8, 2012.

[17] W. F. Enneking and D. A. Campanacci, "Retrieved human allografts. A clinicopathological study," Journal of Bone and Joint Surgery A, vol. 83, no. 7, pp. 971-986, 2001.

[18] D. L. Muscolo, M. A. Ayerza, and L. A. Aponte-Tinao, "Survivorship and radiographic analysis of knee osteoarticular allografts," Clinical Orthopaedics and Related Research, no. 373, pp. 73-79, 2000.

[19] D. L. Muscolo, M. A. Ayerza, L. A. Aponte-Tinao, and M. Ranalletta, "Use of distal femoral osteoarticular allografts in limb salvage surgery," Journal of Bone and Joint Surgery A, vol. 87, no. 11, pp. 2449-2455, 2005. 


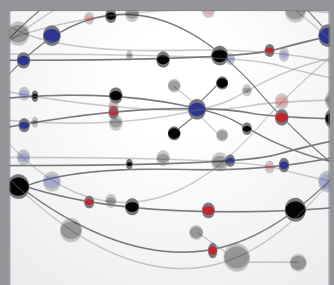

The Scientific World Journal
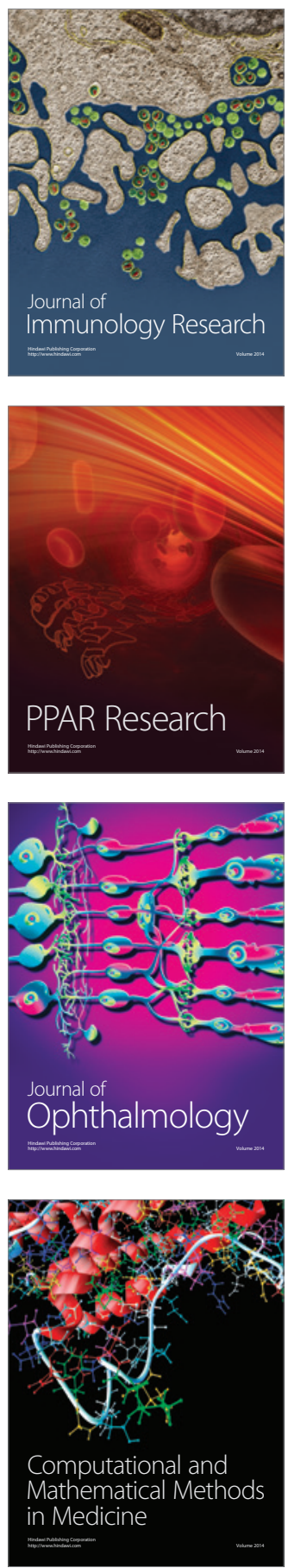

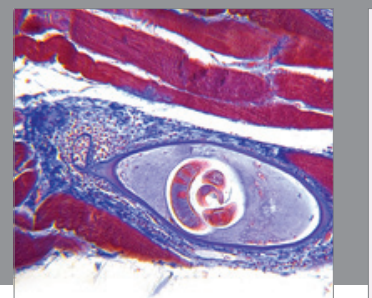

Gastroenterology

Research and Practice
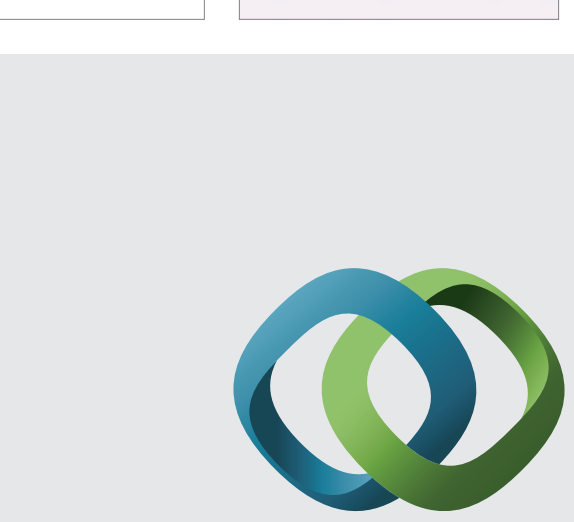

\section{Hindawi}

Submit your manuscripts at

http://www.hindawi.com
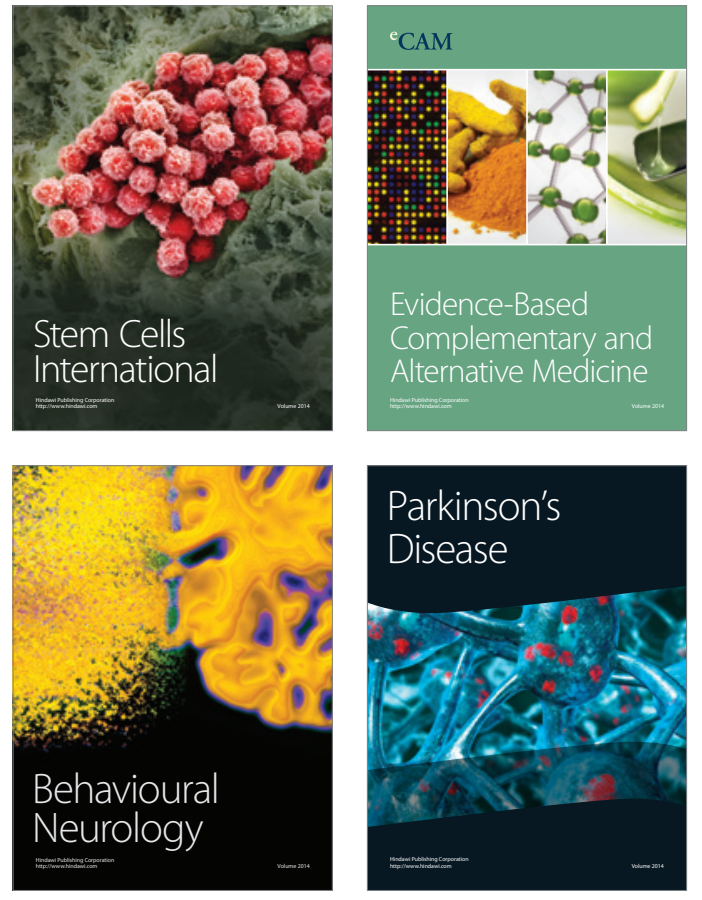
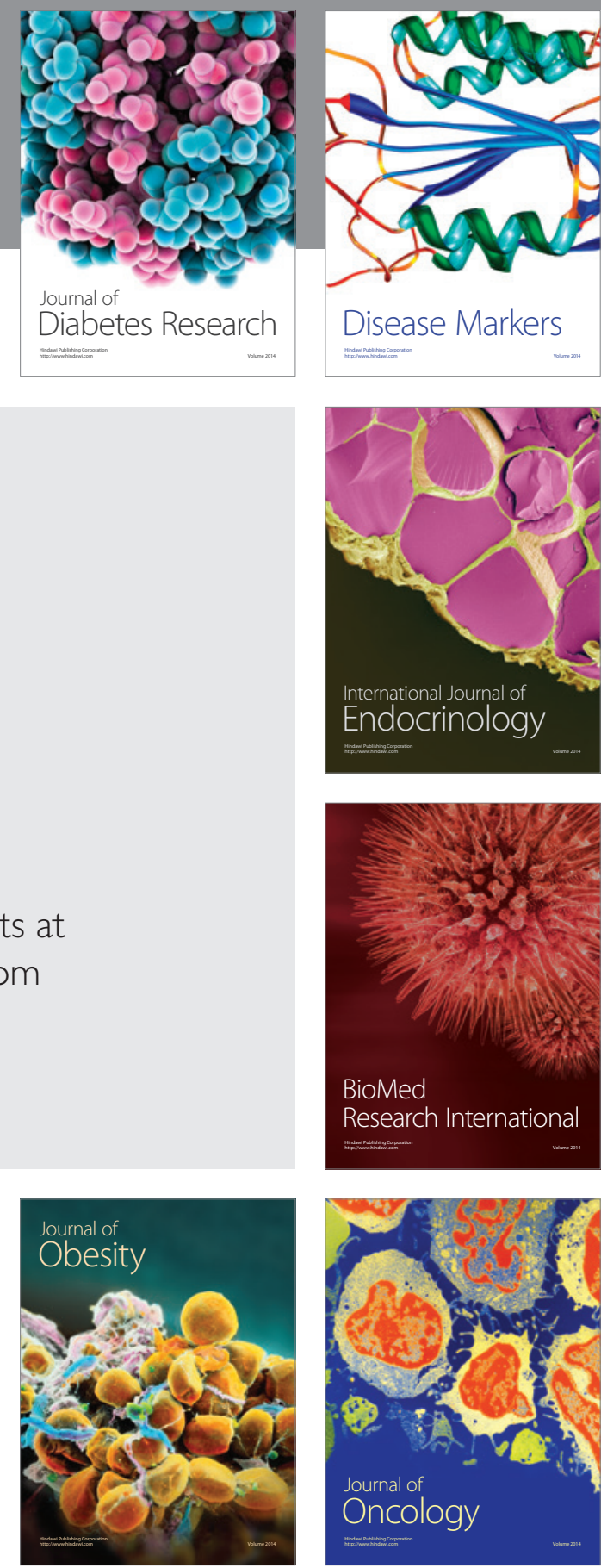

Disease Markers
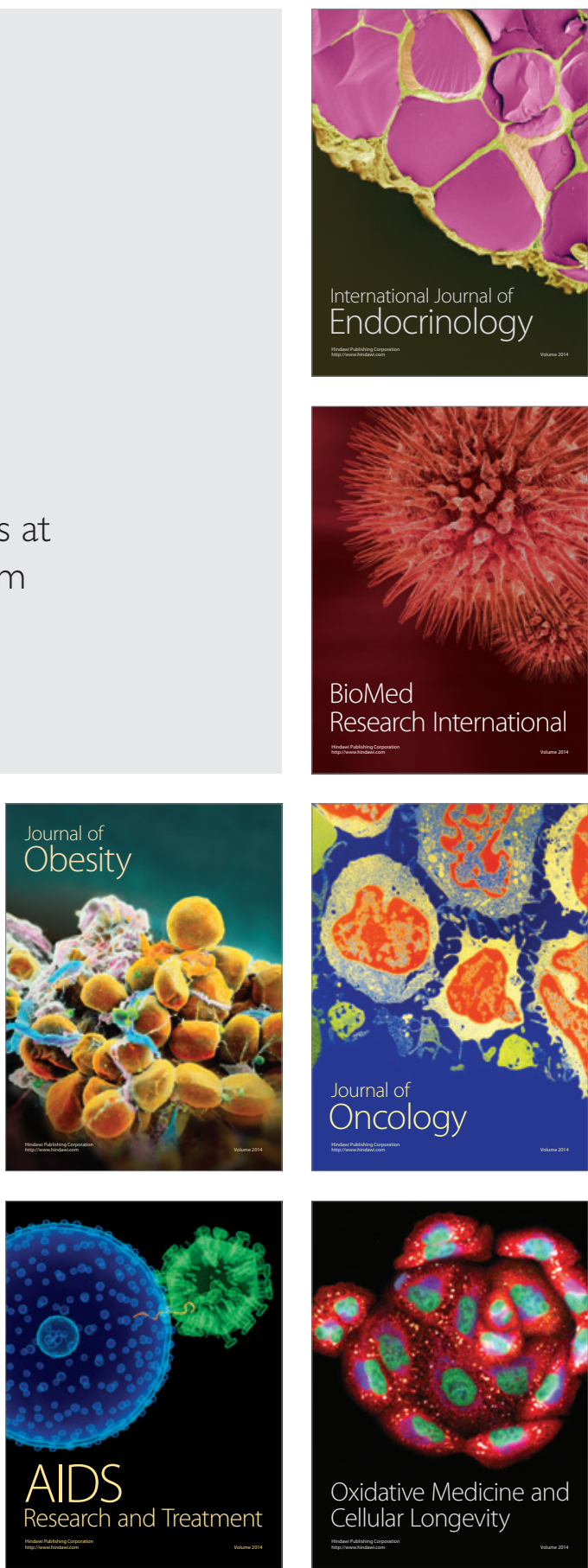